\title{
25 Years of High Definition 3D Scanning: History, State of the Art, Outlook
}

\author{
Bernd Breuckmann \\ Breuckmann $\mathrm{GmbH}$, \\ Torenstr. 14, D-88709 Meersburg, \\ Germany \\ breuckmann-3D@t-online.de
}

\begin{abstract}
After a decade of theoretical approaches, laboratory setups and prototypes it was end of the eighties that the first 3D scanning systems, based on imaging triangulation, were installed for industrial applications, mainly for surface inspection, comparative measurements or deformation analysis. It took another decade to establish 3D scanning in cultural heritage. Nowadays, high definition 3D scanning is state of the art in archaeology and palaeontology, for a wide range of items, from small artefacts like coins, teeth and bones, fragments and scripts up to large figures, statues and small buildings.
\end{abstract}

The paper will start with a short overview on the history of 3D scanning technologies and will discuss in more detail the state of the art of 3D scanners, incl. its current limitations and restrictions. It also will point out the importance of verification and acceptance tests for the data quality of high definition 3D scanners, not only for industrial metrologies but also for most of the applications in arts and cultural heritage. After presenting some extraordinary and challenging projects it will close with an outlook on upcoming and/or expected future developments.

3D scanning. Optical metrology. Fringe projection. Verification and acceptance tests.

\section{INTRODUCTION}

About 30,000 years ago our ancestors started with cave painting (see Figure 1) and manufacturing sculptures and figurines. We do not really know, whether they had cultural or religious reasons, whether they wanted to document their natural environment, in particular animals and their own fellows, or whether they just wanted to explore a new technology. For most of the time of our history, this was the only method to record the real world, however, with the strong intrinsic subjective component of all kind of literature and art.

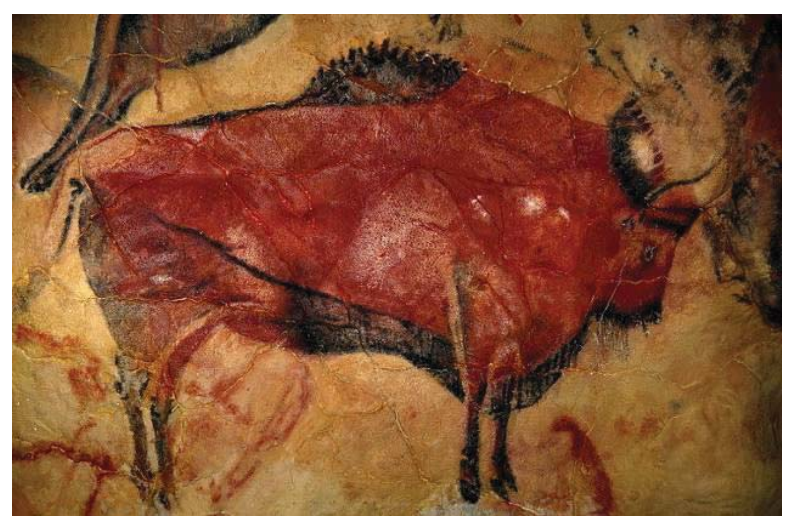

Figure 1: Painting in the Cave of Altamira

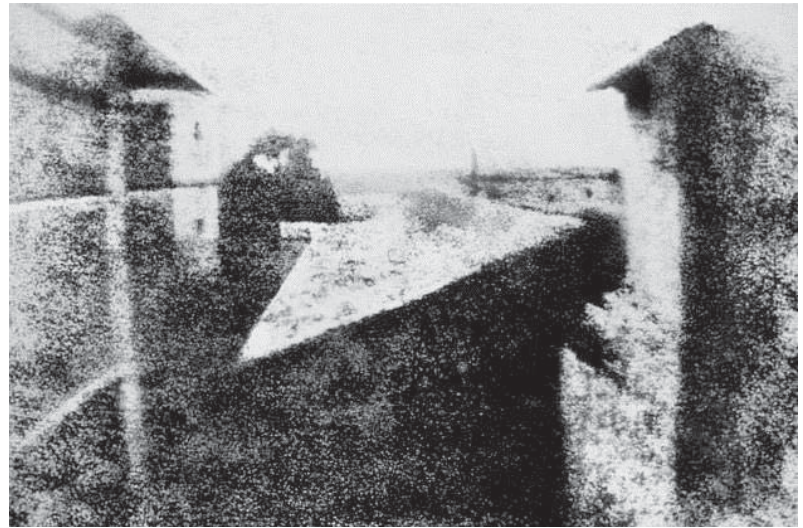

Figure 2: View from the Window at Le Gras, by Joseph Nicéphore Niépce, 1826

The invention of photography in the first half of the 19th century - the first picture was taken in 1826 by Joseph Nicéphore Niépce (see Figure 2) - only less than 200 years ago, provided completely new and more objective possibilities for archiving and documentation and opened the door for a new kind of art. Nevertheless, photography and later on digital image processing, as any kind of 2D recording technique, has strong limitations to reproduce the 3 dimensional world. 


\section{HISTORY OF OPTICAL 3D SCANNING}

5.000 years ago ancient Babylonians and Egyptians have already used triangulation techniques, Euklid and Archimedes have set the mathematical fundamentals of trigonometry about 2.500 years ago, and Snell von Rojen studied the laws of optical triangulation in the 17th century. However, practical 3D scanners, based on triangulation techniques and able to record a 3dimensional picture with sufficient resolution, have been realised only about 25 years ago, after a decade of theoretical approaches, laboratory setups and prototypes and based on the latest developments of image processing systems.

We can assume, that most of the pioneers of the early years, whether they were exploring laser light section techniques as David Addleman in US or topometrical approaches based on fringe projection as the author in Germany, could not completely foresee the potential of this new technology, when they started their developments with analogue video cameras, equipped with camera tubes instead of CCD or CMOS sensors, computers with a CPU (central processor unit) with max. $512 \mathrm{~KB}$, storage devices with a capacity of $5 \mathrm{MB}$ and frame grabbers for converting the optical image into a digitised signal with a typical resolution of $512 \mathrm{x}$ 512 pixel. In particular, the calibration of the scanner with an accuracy and stability, sufficient for metrology applications, was a challenging problem. Therefore, the first 3D scanning systems installed for industrial applications were mainly designed for surface inspection (see Figure 3), comparative measurements or deformation analysis.

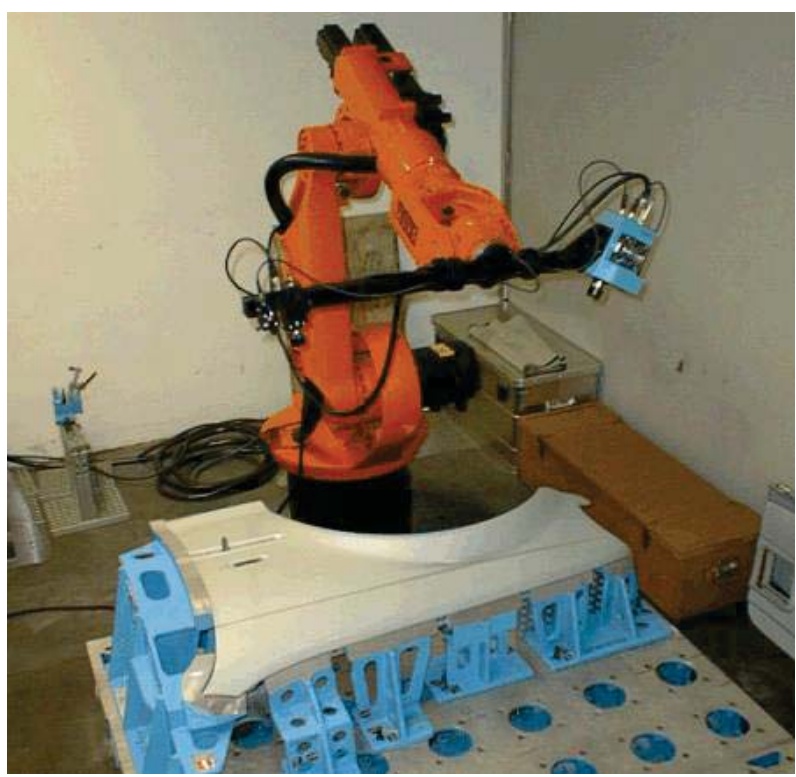

Figure 3: optoSIS Topometrical Surface Inspection System of Breuckmann $\mathrm{GmbH}$
Table 1: Typical specifications of the components of commercial image processing systems

\begin{tabular}{|l|r|r|r|r|}
\hline Year & $\begin{array}{l}\text { CPU I } \\
\text { RAM }\end{array}$ & $\begin{array}{l}\text { Image } \\
\text { memory }\end{array}$ & $\begin{array}{l}\text { HD } \\
\text { capacity }\end{array}$ & $\begin{array}{l}\text { Camera } \\
\text { resolution }\end{array}$ \\
\hline $\mathbf{1 9 8 0}$ & $64 \mathrm{~KB}$ & $512 \mathrm{~KB}\left(^{*}\right)$ & $5 \mathrm{MB}$ & $\left({ }^{* *}\right)$ \\
\hline $\mathbf{1 9 8 5}$ & $512 \mathrm{~KB}$ & $2 \mathrm{MB}\left(^{*}\right)$ & $20 \mathrm{MB}$ & $256 \mathrm{~KB}$ \\
\hline $\mathbf{1 9 9 0}$ & $2 \mathrm{MB}$ & $16 \mathrm{MB}\left(^{*}\right)$ & $100 \mathrm{MB}$ & $1 \mathrm{MB}$ \\
\hline $\mathbf{2 0 0 0}$ & $200 \mathrm{MB}$ & $100 \mathrm{MB}$ & $\mathrm{GB}$ & $4 \mathrm{MB}$ \\
\hline $\mathbf{2 0 1 0}$ & $4 \mathrm{~GB}$ & $2 \mathrm{~GB}$ & $\mathrm{~TB}$ & $8 \mathrm{MB}$ \\
\hline $\mathbf{2 0 1 4}$ & $64 \mathrm{~GB}$ & $32 \mathrm{~GB}$ & $10 \mathrm{~TB}$ & $16 \mathrm{MB}$ \\
\hline
\end{tabular}

$\left.{ }^{*}\right)$ with special memory architecture

$\left.{ }^{* *}\right)$ video cameras with camera tubes

An overview about the developments and the state of the art of the early years is given in (Creath \& Wyant 1992) and (Breuckmann 1993).

\section{STATE OF THE ART OF 3D SCANNERS}

The rapid progress in image processing technology (see table 1), in particular the availability of digital CCD and CMOS cameras with high resolution, allowed the design of 3D scanners for a wide range of applications, in industrial metrology, for medical and dental purposes as well as in arts and cultural heritage. Typical specifications of a high definition topometrical 3D Scanner, based on fringe projection and structured light technique, are shown in Table 2.

Table 2: Typical specifications of the high definition smartSCAN ${ }^{3 D}$ C5 scanner of Breuckmann $\mathrm{GmbH}$

\begin{tabular}{|c|c|}
\hline & smartSCAN $^{\text {3D }}$ C5 \\
\hline Camera type & 2 CCD colour cameras \\
\hline Camera resolution & 5 MPixel, each \\
\hline FOV & 30 to $1500 \mathrm{~mm}$ \\
\hline Operating distance & 250 to $1500 \mathrm{~mm}$ \\
\hline Triangulation angle & $\left(30^{\circ} / 20^{\circ} / 10^{\circ}\right)$ within one \\
\hline Acquisition time & ca. 1 sec $($ fastmode $)$ \\
\hline Sensor weight & ca. $4 \mathrm{~kg}$ \\
\hline X/Y resolution & 10 to $500 \mu \mathrm{m}$ \\
\hline Z resolution & 1 to $50 \mu \mathrm{m}$ \\
\hline
\end{tabular}

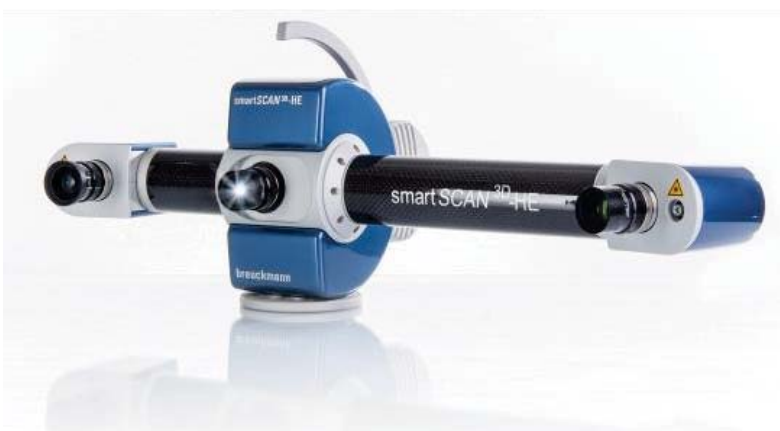

Figure 4: The smartSCAN system of Breuckmann $\mathrm{GmbH}$ 
Since several years, advanced projection devices, based on LCD and DLP technology, allow a more compact design of 3D scanners with increased brightness but reduced power consumption.

\section{NAVIGATION STRATEGIES}

The complete digitisation of a measuring object requires the acquisition of a set of single scans from different viewing positions. For complex objects, hundreds or even thousands of scans might be necessary. The data of the single scans are available in different coordinate systems. To create a merged polygonal mesh, all scan data has to be transformed into a common coordinate system. This data transfer into a common coordinate system is called registration or alignment. State of the art data orientation is achieved by using one of the following measurement and navigation strategies:

- Direct alignment by means of object geometry

- Integrating the scanner or object into a positioning system

- Alignment by means of index marks or reference spheres

- Combination with photogrammetry

- Combination with an optical tracking system

It shall be pointed out, that the alignment strategy might have a strong impact on the overall accuracy of the scan data (see Section 6).

\section{COLOUR AND 3D}

Most applications in cultural heritage require the recording of both $3 \mathrm{D}$ and colour. This can be realised by two different approaches:

- to integrate colour cameras into the 3D scanner

- $\quad$ to map colour images, taken by an external camera, onto the 3D data

Both techniques have special advantages and drawbacks. The first one guarantees a simultaneous and pixel synchronous recording of 3D and colour information; however it requires a constant illumination during the whole scanning process, which might be difficult to realise in particular for large and complex objects. The second one allows to record the colour with a resolution independently from that of the $3 D$ information; but one needs additional equipment and additional steps in the workflow of the data processing.
Independently from the used method, the object is recorded from different viewing directions; thus a very homogenous and diffuse illumination is essential, not only to avoid direct reflections. One also must assure that there are no changes in colour information when recording the same surface area from different viewing directions. Nevertheless, even with the most sophisticated illumination one cannot completely avoid small changes in colour, in particular for surfaces, which are both black and shiny (see Figure 5).

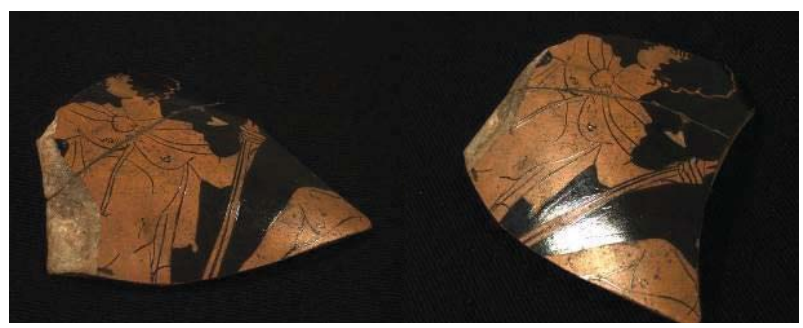

Figure 5: Images of vessel fragment, University Graz, Institute of Archaeology, inv. G 866, recorded from different viewing directions, showing strong reflections and variations in colour

Therefore, combining the different colour information recorded from different viewing points would cause artefacts and colour distortions of the resulting 3D model (see Figure 6a). Advanced software tools are able to detect and correct those reflections and colour changes in the single scans (see Figure 6b). The colour representation recorded and analyzed with this technique is highly comparable to a 2D photography.

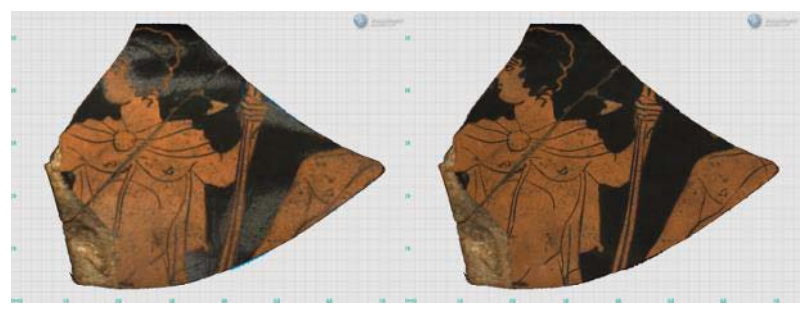

Figure 6: a) Visualisation of the 3D model, analyzed without colour improvement; b) Visualisation of the $3 D$ model, analyzed with a new approach for colour improvement (Breuckmann GmbH, Meersburg)

\section{CORRELATIONS AND LIMITATIONS}

This section will give a brief overview of the correlations between the different parameters of 3D surface scanners and the currently existing limitations.

FOV (Field of View): A larger FOV results in lower $X / Y$ and depth resolution, lower local accuracy, a reduced number of scans and a smaller amount of data. 
Camera resolution: Higher camera resolution results in a better $X / Y$ resolution, or in a larger FOV with the same resolution, along with an increase in the amount of data.

Triangulation angle: Larger triangulation angles result in a better depth resolution and $\mathrm{S} / \mathrm{N}$ ratio, but they may require more scans for complex objects.

Overall accuracy: The choice of the navigation strategy, for example using the alignment by means of the object geometry, a positioning system or photogrammetry as explained in Section 4, has a strong impact on the overall accuracy, not to forget the calibration of the system which is also very essential to achieve high standard accuracy.

Limitations: In addition to all the advantages of topometrical 3D scanners, such as the contact free measurement of a vast number of points within a short space of time and giving access to colour and texture, it always has to be kept in mind that these scanners also have certain limitations. These can be summarised as follows:

- Surface scanning technologies do not give access to volume data

- Triangulation techniques have difficulty in capturing deep holes and undercuttings

- The mandatory principle of structured light techniques is to project light patterns onto the object:

- Due to the limited light sources, it is difficult to illuminate large areas.

- There might be problems in ambient light conditions, especially for large FOV's.

- Problems arising due to shiny and (semi-) transparent surfaces: scanning is possible only with special anti-reflection coatings

- Strong correlation between FOV and resolution

- Typical resolutions are in the $\mu \mathrm{m}$ range (the sub- $\mu \mathrm{m}$ range is difficult to reach)

\section{CERTIFICATION OF OPTICAL 3D SCANNERS}

Topometrical 3D scanners are mainly used as digitisation or measuring device. It is evident that the specifications of a measuring device must be verified at regular intervals, in particular for industrial purposes. However, a lot of users in cultural heritage are not aware of such need or they are not familiar with the corresponding procedures. Many of them trust in the specifications of the manufacturers, sometimes without understanding the correct meaning of the listed parameters. This might be justified, if the digitisation is done just for visualisation and internet presentation. However for documentation, archiving and any kind of measuring purpose it is essential, that the user is verifying the relevant quality parameters, especially the measuring accuracy. The verification of quality parameters requires the following points:

- Definition of quality parameters

- Certified artefacts (see Figure 7)

- Definition of measurement procedure

- Definition of the calculation and interpretation of results

For imaging optical 3D measuring systems providing area-based sampling, the German guidelines VDI/VDE 2634, Part 2 and Part 3, define these practical acceptance tests and re-verification procedures to assess the precision of a system.

For example, the guideline VDI/VDE 2634/2 defines the following Quality Parameters:

- Sphere spacing error

- Probing error

- Flatness measurement error

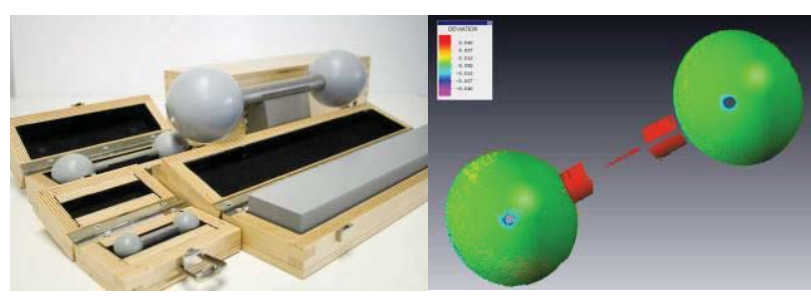

Figure 7: Dumbbell artifact and measurement result

\section{CHALLENGING PROJECTS IN CH}

Within the recent $10-15$ years a large number of challenging projects have been realised, demonstrating that high definition 3D scanners can be used not only in special labs with controlled conditions, but may be used in remote areas and under difficult environmental conditions (Breuckmann 2013).

The objects are documented three-dimensionally under challenging conditions like changing weather, burning sunshine, freezing cold, high humidity, sand and thunder storms as well as dangerous animals.

These applications cover a wide range of objects from very small structures to huge immobile buildings - in different locations - from Europe to Asia and America and even Antarctica, e.g.:

- The David of Michelangelo

- 3D documentation of Mona Lisa, Louvre, Paris

- Deerstones in Mongolia

- The Laocoon in the Vatican Museum

- The Hadrian's Temple in Ephesos 
- Angkor Style Reliefs in Cambodia

- The Terra Nova hut in Antarctica

- Maya Hieroglyphes in Mexico

- The Craftsmanship of Coining

- Footprints in the cave of Pech-Merle

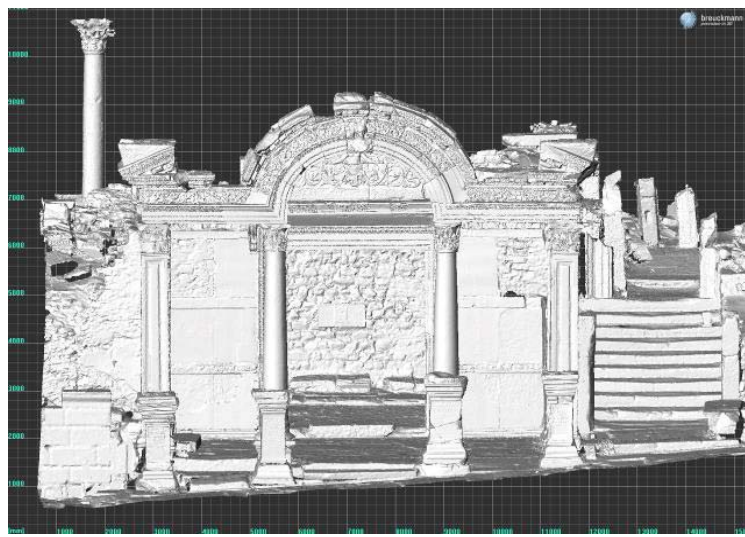

Figure 8: 3D model of the Hadrian's Temple in Ephesus, recorded in 2009 in cooperation with the Austrian Archaeological Institute and the Department of Spatial Development \& Environmental Planning, Vienna University of Technology

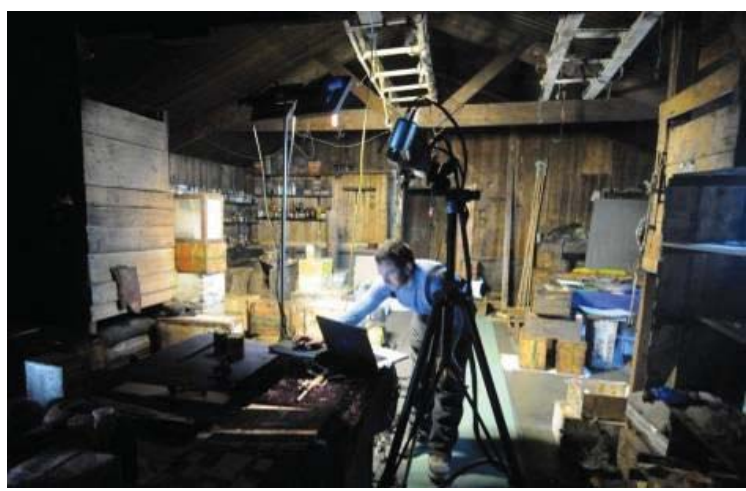

Figure 9: 3D Scanning in the Terra Nova hut in Antarctica, Geometria 2010/2011

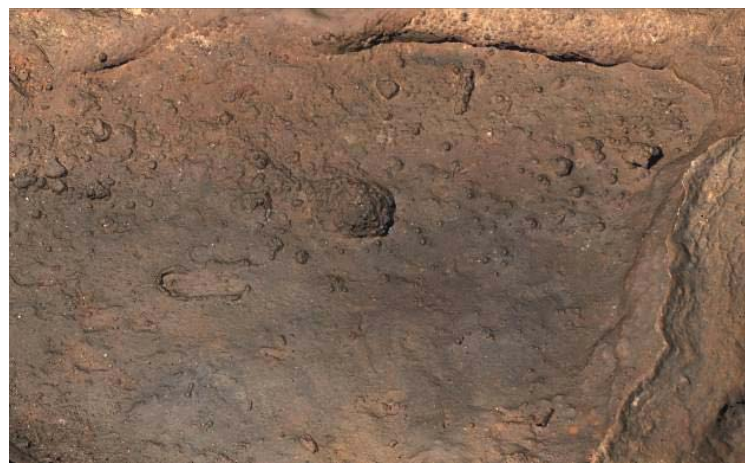

Figure 10: Some 20.000 years old human footprints in the pre-historic cave of Pech-Merle, recorded in 2014 in cooperation with the Neanderthal-Museum, Mettmann

\section{OUTLOOK}

With the increasing use of 3D scanning technology in cultural heritage the prices of the $3 D$ scanners will decrease and the community of users will become more familiar with this technology.

We also can expect a continuous progress in the technical specifications of 3D scanners, e.g. camera resolution, processing power, brightness of the projections units etc. Moreover, there will be faster and advanced algorithms that allow a more automatic processing of even more data in a shorter time. It is also most probable that different scanning technologies as phase shifting technique, the projection of stochastic patterns, multi-viewstereometry and others will grow together, increasing the flexibility and versatility of the scanning equipment, in particular for those applications where the scanner is coming to the object of interest. Another solution will be designed for mass digitisation of collections. In this case the scanning equipment will include positioning devices, e.g., a robotic articulation, which will result in a much larger, less flexible and transportable setup; thus, the objects must be brought to the scanner.

\section{REFERENCES}

Creath, K., and Wyant, J. C. (1992) Moiré and fringe projection techniques, chapter 16, pp. 653685. In D. Malacara (ed.), Optical Shop Testing, John Wiley \& Sons.

Breuckmann, B. (1993) Bildverarbeitung und Optische Messtechnik in der industriellen Praxis, Franzis Verlag, München.

Breuckmann, B. (2013) Challenging 3D applications in arts and cultural heritage, EVA Florence 2013. 